\title{
Identificação de demandas para atendimento e implantaçáo do serviço de Terapia Ocupacional em um hospital universitário
}

\author{
Miryam Bonadiu Pelosi, Janaína Santos Nascimento \\ Departamento de Terapia Ocupacional, Universidade Federal do Rio de Janeiro - UFRJ, \\ Rio de Janeiro, RJ, Brasil.
}

\begin{abstract}
Resumo: Introdução: No contexto hospitalar, o terapeuta ocupacional atua como promotor da saúde e da qualidade da vida ocupacional. Para que o serviço de Terapia Ocupacional seja criado em um hospital, pode ser necessário adotar e articular diferentes estratégias. Objetivo: Caracterizar tanto as principais solicitações como os atendimentos terapêuticos, desenvolvidos pelas residentes de Terapia Ocupacional no processo de análise de demanda para a implantação do serviço. Método: Pesquisa com abordagem quanti-qualitativa. Analisaram-se 151 pedidos de avaliação recebidos para o atendimento de Terapia Ocupacional dos pacientes atendidos em um hospital universitário, no período de junho de 2012 a junho de 2013. Foram realizadas as análises de conteúdo e descritiva. Resultados: No decorrer do período deste estudo, a Terapia Ocupacional desenvolveu ações que envolveram os pacientes hospitalizados, os seus cuidadores e a equipe. Os pacientes encaminhados para o atendimento tinham idade entre 14 e 88 anos, e apresentavam dificuldades principalmente relacionadas à regulação emocional e ao processo de hospitalização. Os atendimentos foram feitos por meio de abordagens individuais e em grupo; dentre esses atendimentos, os mais efetuados foram de acolhimento e orientação para o paciente, familiar, o cuidador e o profissional. Conclusão: Os resultados deste estudo agregam conhecimento aos fatores relacionados à análise da demanda para atuação da Terapia Ocupacional hospitalar e reforçam a necessidade de esses profissionais atentarem para os requisitos imprescindíveis à implantação de serviços.
\end{abstract}

Palavras-chave: Terapia Ocupacional, Atividades Cotidianas, Assistência Hospitalar.

\section{Identification of service demand and Occupational Therapy service implementation in a university hospital}

\begin{abstract}
Introduction: The occupational therapist acts as a promoter of health and quality of occupational life in the hospital context. In order to implement the Occupational Therapy Service in a hospital, it may need to adopt and articulate different strategies. Objective: To characterize the main requests and therapeutic services developed by the Occupational Therapy residents during the demand analysis process to implement the service. Method: This is a quantitative and qualitative research approach. We analyzed 151 assessment requests received for the Occupational Therapy service of patients treated at a university hospital, from June 2012 to June 2013. Data were submitted to descriptive and content analysis. Results: During the study period, the Occupational Therapy developed actions involving hospitalized patients, their caregivers and staff. Patients referred for care were aged between 14 and 88 years and had difficulties mainly related to emotional regulation and the hospitalization process. The service was provided through individual and group approaches, and among them, the most requested were welcoming and orientation to the patient, family, caregivers and professional. Conclusion: The results of this study add knowledge to the factors related to the demand analysis for of Occupational Therapy and reinforce the need for these professionals focus their attention on the requirements essential to implement the services.
\end{abstract}

Keywords: Occupational Therapy, Activities of Daily Living, Hospital Care.

Autor para correspondência: Miryam Bonadiu Pelosi, Coordenação do Curso de Terapia Ocupacional, Rua Prof. Rodolpho Paulo Rocco, s/n, Prédio do Centro de Ciências da Saúde, Bloco K, Sala 17, Cidade Universitária, CEP 21910-590, Ilha do Fundão, Rio de Janeiro, RJ, Brasil, e-mail: miryam.pelosi@gmail.com

Recebido em Set. 15, 2015; $1^{\text {a }}$ Revisão em Dez. 20, 2015; $2^{\text {a }}$ Revisão em Fev. 17, 2016; 3ª Revisão em Abr. 6, 2016 ; Aceito em Maio 19 , 2016. 


\section{Introdução}

A rotina hospitalar envolve condiçôes de permanência que são marcadas por regras e horários preestabelecidos e cuidados direcionados ao tratamento da doença. Neste contexto, as atividades possíveis de serem realizadas, como tomar banho, ir ao banheiro e se alimentar, são organizadas em rotinas rígidas, que contribuem para a perda do controle do indivíduo sobre a própria vida e do respeito aos seus desejos (ANGELI; LUVIZARO; GALHEIGO, 2012).

Além disso, fatores relacionados às limitaçôes ou às incapacidades anteriores ao processo de internação, ocasionados pela doença e suas consequências, somados às restriçôes e aos cuidados exigidos no seu tratamento, influenciam o desempenho ocupacional no ambiente hospitalar (SILVEIRA; JOAQUIM; CRUZ, 2012; SANTOS; DE CARLO, 2013).

Ressalta-se que as instituiçóes hospitalares são locais que possuem características comuns. Muitas têm enfermarias e Centros de Terapia Intensiva coletivos, ausência de armários para guardar os pertences do paciente ou restrição de equipamentos, o que faz com que o hospital seja um local muito diferente do ambiente domiciliar.

Verifica-se, assim, que múltiplos fatores podem influenciar o desempenho ocupacional do indivíduo e, consequentemente, a manutençáo dos seus papéis ocupacionais, o que reforça a necessidade de um terapeuta ocupacional no contexto hospitalar como membro da equipe.

Para o terapeuta ocupacional, o envolvimento ativo dos indivíduos em ocupaçôes promove, facilita, apoia e favorece a manutenção da saúde, do bem-estar e do desenvolvimento de atividades que os próprios pacientes consideram fundamentais em sua vida (AMERICAN..., 2014).

Neste sentido, no contexto hospitalar, o terapeuta ocupacional atua como promotor da saúde e da qualidade da vida ocupacional - tanto durante como após a internação (DE CARLO et al., 2006) - e utiliza ocupaçóes e atividades selecionadas de forma terapêutica como métodos primários de intervenção em todo o processo (AMERICAN..., 2014). O trabalho desse profissional inclui também a atenção aos cuidadores formais e informais, à equipe assistencial e com o ambiente hospitalar.

Santos e De Carlo (2013) verificaram, a partir de uma revisáo da literatura sobre o trabalho dos terapeutas ocupacionais no contexto hospitalar, que os profissionais dessa área possibilitam vivências para os pacientes e seus familiares, as quais promovem o enfretamento da hospitalizaçáo e do adoecimento, por meio da realização de afazeres saudáveis e do resgate ou da descoberta de novas capacidades e habilidades, além de criar condições para a manutenção da vida cotidiana.

Para que o terapeuta ocupacional trabalhe nesse contexto, ele necessita do conhecimento técnico-científico e do histórico cultural do ambiente em que seu trabalho está inserido (SANTOS; DE CARLO, 2013). Devem-se também considerar os diferentes espaços e programas hospitalares, além dos aspectos relacionados às características do processo de adoecimento e hospitalização, tais como o conhecimento acerca da doença e suas complicaçôes, implicaçóes emocionais e sociais, e estratégias de enfrentamento (DE CARLO et al., 2006). Este conhecimento possibilita ao profissional elaborar e implantar estratégias de ação sensíveis e pertinentes, que contemplem as necessidades das pessoas atendidas (GALHEIGO; ANGELI, 2008).

Além disso, torna-se importante que haja um setor de Terapia Ocupacional e profissionais da área, favorecendo a análise da demanda e o desenvolvimento de um programa terapêutico-ocupacional. Estudo conduzido por terapeutas ocupacionais da Universidade de São Paulo descreveu a importância desse processo para a implantação do serviço e ressaltou a necessidade de valorização de seu papel no contexto hospitalar (DE CARLO et al., 2006).

\section{Identificação de Demandas para Atendimento e Implantação do Serviço de
Terapia Ocupacional em Um
Hospital Universitário}

O hospital universitário, local desta pesquisa, é um dos espaços de assistência de uma universidade federal da Região Sudeste do país, que teve o trabalho da Terapia Ocupacional impulsionado pelo início da graduação em Terapia Ocupacional em 2009, pela Residência Integrada Multiprofissional em Saúde - que disponibilizou duas vagas para esse profissional em 2011 - e por projetos de pesquisa e extensão conduzidos por docentes do curso, com início neste mesmo ano.

A estrutura física do referido hospital é composta por 13 andares e abriga diversas clínicas, como Clínica de Cardiologia, Centros de Tratamento Intensivo, Clínica da Dor e Cuidados Paliativos, Clínica Médica, Geriatria, Oncologia/Hematologia, Neurologia, Ortopedia, dentre outras. Trata-se de 
um hospital de atendimento para jovens a partir de 14 anos e adultos.

Até a realização deste estudo, a área de atuação da Terapia Ocupacional era pouco conhecida pelos demais profissionais do hospital, assim como os diversos setores e serviços já existentes no hospital eram, também, pouco familiares aos residentes e seus preceptores. Por essa razão, os espaços para intervenção e ensino-aprendizado da Terapia Ocupacional precisaram ser conquistados pelos residentes e seus supervisores, ou seja, as três professoras efetivas do Departamento de Terapia Ocupacional da universidade que atuavam no contexto hospitalar.

A princípio, realizou-se um estudo de campo em diferentes locais do hospital com o objetivo de apresentar a Terapia Ocupacional, analisar as demandas, estabelecer parcerias e traçar metas e critérios para o desenvolvimento dos atendimentos, além de divulgar a profissão, seu campo de atuação e seu papel no contexto hospitalar. Antes do atendimento profissional, foram realizadas palestras e distribuídos folhetos informativos para as equipes. Além disso, foi fixado nos murais de cada clínica o contato das residentes da área.

Os atendimentos da Terapia Ocupacional aconteciam mediante solicitação e/ou recebimento de um pedido de avaliação feito pelo médico responsável pelo paciente. Esta dinâmica de encaminhamento, pautada no modelo biomédico que caracterizava a micropolítica do hospital, protelava, em vários momentos, o trabalho da Terapia Ocupacional, pois, mesmo quando os residentes identificavam a necessidade de atendimento terapêutico-ocupacional, era preciso solicitar um parecer médico para iniciar o trabalho.

Em outros hospitais, o trabalho da Terapia Ocupacional na enfermaria também tem início a partir do recebimento de pedidos de interconsultas. No entanto, esses encaminhamentos podem ser provenientes de diversos profissionais de Saúde, como fisioterapeutas, enfermeiros, fonoaudiólogos, psicólogos ou mesmo terapeutas ocupacionais, e não apenas dos médicos (MARTINS et al., 2009; PENGO, 2012).

No início, os pedidos para avaliação da Terapia Ocupacional eram fixados nos murais das clínicas ou deixados, soltos, nas bancadas das recepçốes dos diferentes setores. A ausência de um local de referência da Terapia Ocupacional dificultou a resposta aos pedidos de avaliaçáo, provocando a perda ou a resposta tardia de alguns pareceres.

Para minimizar o problema, os preceptores fixaram suportes de acrílico nas diversas clínicas com a identificação "Pedidos de avaliação para a Terapia Ocupacional" e os residentes, então, passaram a fazer uma busca cuidadosa nesses locais.

Na medida em que não havia terapeutas ocupacionais contratados ou concursados, as quatro residentes recebiam pedidos de avaliação de todo o hospital e as preceptoras precisavam conciliar a supervisão das residentes com as demais atividades acadêmicas do curso de graduação em Terapia Ocupacional.

As residentes atendiam pacientes jovens e adultos, acometidos por diferentes patologias e internados nas mais diversas unidades ou enfermarias. O tempo de acompanhamento dos casos variava entre internaçóes curtas e internaçóes muito longas, agravadas por problemas sociais, como a ausência de um familiar para se responsabilizar pela alta ou a falta de condiçóes para a continuidade do tratamento em nível domiciliar.

Destaca-se que a introdução do serviço de Terapia Ocupacional, a partir do Programa de Residência e com o apoio dos professores do curso de graduaçáo em Terapia Ocupacional, foi o ponto de partida desse trabalho na área hospitalar.

Em 2011, a equipe de Terapia Ocupacional conseguiu que seu símbolo fosse incluído no ProntiHU, sistema de prontuário eletrônico, para que esta pudesse responder aos pedidos de avaliação encaminhados à área e registrar as evoluções dos atendimentos. Antes, as respostas eram realizadas no espaço destinado a todos os profissionais de Saúde, o que dificultava a localização das evoluçôes da Terapia Ocupacional.

Neste mesmo ano, diante do leque de possibilidades de atuação do profissional nesse hospital, iniciou-se o processo de regulamentaçáo do serviço de Terapia Ocupacional finalizado em 2016.

No ano de 2012, uma sala de atendimento ambulatorial no térreo do hospital foi conquistada, ao lado de ambulatórios de outras especialidades. Em 2014, ocorreu a abertura de concurso para a contratação de cinco terapeutas ocupacionais para o Departamento de Terapia Ocupacional e um profissional foi alocado no hospital universitário no ano seguinte. Atualmente, este hospital é importante campo de estágio para os alunos da graduação do curso de Terapia Ocupacional, sob supervisão dos docentes.

Muitas estratégias foram utilizadas para implantação do serviço nesta área, entre as quais a análise de demanda de atendimento em Terapia Ocupacional no contexto hospitalar.

O objetivo deste estudo foi caracterizar as principais solicitações e os atendimentos terapêuticos 
desenvolvidos pelas residentes de Terapia Ocupacional no processo de análise de demanda para a implantação do serviço, a partir dos encaminhamentos médicos.

\section{Método}

Trata-se de uma pesquisa com abordagem quanti-qualitativa e retrospectiva ${ }^{1}$, desenvolvida em um hospital universitário da Região Sudeste do país.

Para fins do estudo, compuseram a amostra 151 pedidos de avaliação recebidos para o atendimento de Terapia Ocupacional no período de junho de 2012 a junho de 2013. Dentre estes, 43 foram excluídos em virtude de não terem sido respondidos por razóes como óbito ou a alta do paciente antes da possibilidade de resposta, ou pela impossibilidade de atender a demanda do hospital, com a equipe de residentes disponível. Portanto, foram avaliados 108 pedidos de avaliação.

Os atendimentos foram realizados por quatro residentes de Terapia Ocupacional, duas do primeiro ano de residência e, as outras, do segundo, supervisionadas por professores do curso de Terapia Ocupacional.

Os dados dos pacientes foram obtidos a partir da análise dos pedidos médicos de avaliação terapêutica-ocupacional encaminhados às residentes, das respostas a essas solicitaçôes e de suas evoluçôes.

Ressalta-se que as informaçôes contidas nesses pedidos eram: data de elaboração do documento; localizaçáo do paciente; nome do médico que realizou a solicitação e o motivo, e a resposta do terapeuta ocupacional a quem o pedido fora encaminhado.

Para a avaliação dos documentos, utilizou-se a análise de conteúdo organizada em três etapas: pré-análise, exploração do material e tratamento dos resultados, envolvendo a inferência e a interpretação (BARDIN, 2009). A partir desta análise, foram construídas categorias relacionadas às principais dificuldades apresentadas pelos pacientes, bem como para as respostas dos terapeutas ocupacionais aos pedidos e suas açóes.

Posteriormente, elaborou-se o banco de dados em planilha do Excel $^{\circ}$ com a dupla digitação das categorias. Para a análise dos dados, o banco de dados foi importado para o software Statistical Package for The Social Sciences - SPSS, versão 17.0. Por fim, os dados foram submetidos à estatística descritiva, utilizando-se frequências relativas para as variáveis categóricas e, para as numéricas, medidas de tendência central (média ou mediana).
O projeto foi aprovado pelo Comitê de Ética em Pesquisa com Seres Humanos do HUCFF/UFRJ, sob o Protocolo n ${ }^{\circ}$ 66/2011.

\section{Resultados e Discussão}

\subsection{Perfil dos pacientes atendidos pelos terapeutas ocupacionais}

No presente estudo, a população encaminhada para atendimento em Terapia Ocupacional é composta por pessoas com idade entre 14 e 88 anos, com média de 54,6 anos, sendo que aproximadamente $90 \%(\mathrm{~N}=96)$ da amostra tinha mais de 30 anos. O grupo mais representativo foi o de idosos acima de 60 anos, que compuseram $46 \%(\mathrm{~N}=50)$ da amostra, corroborando com pesquisa realizada em uma unidade de Niterói-RJ, cujos resultados apontaram para um maior percentual de internaçóes de idosos na faixa etária de 70 a 79 anos (TENÓRIO; CAMACHO, 2015).

Este aspecto pode estar relacionado às modificaçôes e ao declínio em múltiplos sistemas decorrentes da interação de mecanismos fisiológicos do envelhecimento natural e sua associação com o aparecimento de multimorbidade, que contribuem para a perda da capacidade adaptativa e para maior chance de déficits na saúde (ESQUENAZI; SILVA; GUIMARÃES, 2014).

Quanto ao sexo, $54 \%(\mathrm{~N}=58)$ dos pedidos de avaliação foram relativos a encaminhamento de pacientes do sexo feminino e $46 \%(\mathrm{~N}=50)$, do sexo masculino. Dados semelhantes foram encontrados em pesquisas conduzidas no hospital de Montes Claros-MG (RABELO et al., 2010) e em uma unidade hospitalar do município de Niterói-RJ, em que a maioria dos pacientes era do sexo feminino (TENÓRIO; CAMACHO, 2015). Este fato pode ser justificado em virtude de as mulheres serem a maioria da população brasileira $(50,77 \%)$ e as principais usuárias do Sistema Único de Saúde (SUS) (BRASIL, 2011).

A maior parte dos atendimentos da Terapia Ocupacional aconteceu nas enfermarias $d a$ Oncologia/Hematologia, 29\% ( $\mathrm{N}=32)$, da Clínica Médica, $28 \%(\mathrm{~N}=30)$, e da Cardiologia, $21 \%$ $(\mathrm{N}=23)$.

Um estudo de revisão relacionado às açóes dos terapeutas ocupacionais, com a população adulta e idosa, no período de 1990 a 2006, mostrou que a atuação desses profissionais vinha acontecendo principalmente nas enfermarias de Clínica Médica (Cardiologia, Endocrinologia, Gastroenterologia, 
Geriatria, Neurologia, Nefrologia, Oncologia, Pneumologia e Reumatologia), na Clínica Cirúrgica e na de Doenças Infecciosas (GALHEIGO, 2007).

No que se referem às patologias mais frequentemente assinaladas nos pedidos de avaliação, os maiores percentuais foram de doenças cardíacas, $37 \%(\mathrm{~N}=40)$, seguidas das oncológicas e hematológicas, 29\% $(\mathrm{N}=31)$, e as neurológicas, $11 \%(\mathrm{~N}=12)$. Foram sinalizados, ainda, os problemas respiratórios, as doenças infectocontagiosas e as reumatológicas, com $3 \%$ cada um desses quadros $(\mathrm{N}=3)$. A população com mais de uma dessas patologias citadas representou $14 \%(\mathrm{~N}=15)$.

Semelhantes aos dados obtidos neste estudo, outras pesquisas verificaram que a atuação da Terapia Ocupacional, de forma geral, vem ocorrendo com pacientes acometidos por patologias diversas, independentemente se o problema é agudo, crônico ou em fase de exacerbação ou agudização de uma doença crônica (BORGESA; LEONIA; COUTINO, 2012; DE CARLO et al., 2006).

\subsection{Principais dificuldades apresentadas pelos pacientes}

A partir da análise das respostas dos pedidos de avaliação encaminhados aos residentes de Terapia Ocupacional, foram organizadas as seguintes categorias: dificuldades relativas ao processo de hospitalização; habilidades percepto-sensoriais; habilidades práxica e motora; adaptação emocional; habilidades cognitivas; habilidades sociais e de comunicação. Os principais obstáculos estavam relacionados aos aspectos emocionais, $70 \%(\mathrm{~N}=76)$, e ao processo de hospitalização, $65 \%(\mathrm{~N}=70)$. Ressalta-se que, não raramente, os profissionais responderam aos pedidos de avaliação sinalizando mais de uma demanda de trabalho a ser realizado.

Dentre as dificuldades emocionais, os principais fatores referidos pelos pacientes foram: isolamento social; dependência; sentimentos de medo; desconforto; angústia; preocupaçôes, e dúvidas, que se acentuavam quando eles desconheciam ou não tinham clareza do seu diagnóstico, dos problemas relacionados a ele ou sobre o tempo de internação. Essas questóes eram manifestadas pelos pacientes por meio de alteraçóes de humor e comportamento, problemas de interaçáo, de participação no tratamento e no desempenho das ocupaçóes.

No que se refere ao processo de hospitalização, os pacientes relataram dificuldades em lidar com: o afastamento e as mudanças dos seus papéis ocupacionais; as barreiras à participação em ocupações significativas; a perda da referência do seu espaço, e a perda de controle sobre a própria vida e seus desejos.

Santos e De Carlo (2013) sinalizam que o tratamento hospitalar apresenta características muito diversas da rotina do paciente, o que provoca uma ruptura no cotidiano dele e dos familiares envolvidos no seu cuidado.

Apesar de todos os pacientes hospitalizados conviverem com situação semelhante, ressalta-se que alguns deles conseguiam apresentar estratégias de enfrentamento para lidar com as dificuldades impostas pela hospitalizaçáo e pelo adoecimento. As mais observadas neste estudo foram: a busca para se manter ativo durante o período de internação e estratégias relacionadas à religiáo, à espiritualidade e às crenças pessoais.

\subsection{Caracterização dos atendimentos da Terapia Ocupacional no hospital}

A partir da análise dos documentos relacionados aos atendimentos de Terapia Ocupacional, foram organizados em categorias os recursos mais utilizados pelos profissionais e/ou solicitados pelos pacientes: acolhimento e orientaçóes para o paciente, o familiar, o cuidador e o profissional, $82 \%(\mathrm{~N}=88)$; atividades de lazer que envolviam a possibilidade de ouvir músicas, ler, pintar, jogar e conversar sobre diferentes assuntos, como novelas e esportes, $67 \%$ $(\mathrm{N}=72)$; conforto espiritual, $63 \%(\mathrm{~N}=68)$; apoio a atividades de vida diária e organização da rotina ocupacional, $55 \%(\mathrm{~N}=59)$, e recursos e estratégias para favorecer a Comunicaçáo Alternativa, 35\% $(\mathrm{N}=37)$.

No decorrer dos atendimentos relatados, realizados por meio de abordagens individuais e em grupo, a Terapia Ocupacional desenvolveu açóes com os pacientes hospitalizados, os seus cuidadores, formais ou informais, e a equipe.

Os principais objetivos do trabalho do terapeuta ocupacional foram: treinar, orientar, adaptar e/ou criar estratégias para melhorar o desempenho das atividades; resgatar atividades prazerosas do contexto do paciente; auxiliar e instrumentalizar o paciente na elaboração e na conclusão de projetos de vida; trabalhar o luto a partir da busca de reconstrução de significados; desenvolver açôes de natureza interdisciplinar em conjunto com outros profissionais envolvidos nos cuidados, como, por exemplo, na comunicação de más notícias.

Nas clínicas em que ocorreu a maior parte dos atendimentos, os procedimentos realizados foram: 
orientaçôes e acolhimento; resgate de atividades que tinham significado e valor ao paciente; açóes de ambientação e humanização; atividades funcionais; preparação pré e pós-cirúrgica, e orientação para programação de alta.

Ressalta-se que os acompanhamentos desenvolvidos consideravam as preferências e os desejos dos pacientes, mas também as suas necessidades terapêuticas; a idade; o nível de alerta; a situação clínica e psicossocial do paciente no momento da intervenção; as suas habilidades; as exigências, e os cuidados necessários para a prevenção e o controle das infecçóes hospitalares.

Os atendimentos de orientação aos pacientes e familiares incluíram estruturação e organização da rotina do paciente, bem como simplificaçáo, adaptação e treino das atividades em que eles apresentaram dificuldades.

A respeito do conforto espiritual, as ações relacionadas envolveram a criação de espaços para a discussão das diferentes formas de se relacionar consigo mesmo, com o outro, com a natureza, com Deus e com o Universo, além da possibilidade de realizar atividades, como preces, leitura de livros sagrados e atividades de completar ou procurar palavras que tivessem relação com temas da religiáo do paciente.

Os atendimentos de apoio incluíram confecção de órteses e objetos adaptados para posicionamento e/ou promoção da independência funcional/ocupacional.

Quanto à Comunicação Alternativa, as açôes compreenderam a facilitação da comunicação oral e escrita, por meio do uso de recursos de baixa tecnologia, como pranchas de comunicação e pranchetas para escrever, e de recursos de alta tecnologia, como comunicadores, tablets, computadores, bem como a utilização do celular e das redes sociais para a comunicação com familiares e amigos.

Em estudo realizado com 34 terapeutas ocupacionais que atuavam em hospitais na capital baiana, constatou-se que as principais intervençóes realizadas estavam relacionadas à avaliação e à orientação no contexto hospitalar, à utilização da Tecnologia Assistiva, ao treino de Atividades da Vida Diária (AVD) e à intervenção cognitiva (BORGESA; LEONIA; COUTINO, 2012).

Além disso, foram oferecidos momentos de atenção aos profissionais de saúde, com o intuito de melhorar a qualidade da interação entre a equipe e os pacientes, e criar oportunidade de lazer, descontraçáo e um espaço de escuta para questóes inerentes ao ato de cuidar. Dentre estas açóes, ressalta-se o grupo "Cuidando de quem cuida", criado em parceria com a psicologia para a equipe de enfermagem, com frequência semanal. Organizou-se um espaço acolhedor, de troca de saberes e apoio mútuo, no qual os profissionais puderam trazer questóes inerentes ao ato de cuidar, os seus limites e as dificuldades ocasionadas pelos afetos e desafetos desenvolvidos nas relaçóes com os pacientes e também pelo luto provocado pelas perdas vivenciadas no trabalho.

Como resultado, verificou-se que a atuação da Terapia Ocupacional possibilitou benefícios para a rotina ocupacional dos pacientes atendidos, bem como dos profissionais, contribuindo para a melhora da qualidade de vida e do desempenho ocupacional.

\section{Conclusão}

A análise de demanda do atendimento de Terapia Ocupacional no hospital teve como ponto de partida a residência multiprofissional e o apoio dos professores do curso de graduação em Terapia Ocupacional, responsáveis pelas supervisões e pelo desenvolvimento de projetos de pesquisas e extensão na área de contexto hospitalar.

As principais dificuldades nesse processo estiveram relacionadas ao desconhecimento da área de atuação da Terapia Ocupacional pelos demais profissionais e estudantes do hospital. E, de forma recíproca, pelo fato de os diversos setores e serviços já existentes no hospital serem, também, pouco familiares aos residentes de Terapia Ocupacional e seus preceptores.

Desse modo, para que a implantação se efetivasse e fosse possível a análise dessa demanda, houve a necessidade de estudo de campo em diferentes espaços do hospital; a apresentação da profissão e dos objetivos do atendimento realizado pela terapeuta ocupacional às equipes clínicas; a divulgação por meio de folhetos informativos, e a inserção do terapeuta ocupacional nas reunióes de equipe para auxiliar no processo de encaminhamento, análise e manutenção da demanda.

Após a avaliação dos documentos que registraram os atendimentos de Terapia Ocupacional, foi possível elencar o repertório de atividades e recursos mais utilizados como instrumentos de trabalho do terapeuta ocupacional na implantação do serviço nesse hospital universitário.

Os resultados deste estudo agregam conhecimento aos fatores que estáo relacionados à análise da demanda para atuação da Terapia Ocupacional e reforçam a necessidade de esses profissionais estarem atentos aos requisitos imprescindíveis à implantação de serviços.

\section{Referências}


AMERICAN OCCUPATIONAL THERAPY ASSOCIATION - AOTA. Occupational Therapy practice framework: domain and process. American Journal of Occupational Therapy, Rockville, v. 68, n. 6, p. 1-43, 2014.

ANGELI, A. A. C.; LUVIZARO, N. A.; GALHEIGO, S. M. O cotidiano, o lúdico e as redes relacionais: a artesania do cuidar em terapia ocupacional no hospital. Interface - Saúde, Educą̧ão, Comunicação, Botucatu, v. 16, n. 40, p. 261-271, 2012.

BARDIN, L. Análise de conteúdo. Lisboa: Edições 70, 2009.

BORGESA, F.; LEONIA, T. F.; COUTINO, I. Terapia Ocupacional no contexto hospitalar: um delineamento da profissão em hospitais gerais e especializados na cidade de Salvador, BA. Cadernos de Terapia Ocupacional da UFSCar, São Carlos, v. 20, n. 3, p. 425-433, 2012.

BRASIL. Ministério da Saúde. Secretaria de Atenção à Saúde. Departamento de Açôes Programáticas Estratégicas. Politica Nacional de atenção integral à saúde da mulher: princípios e diretrizes. Brasília: MS, 2011.

DE CARLO, M. M. R. P. et al. Terapia Ocupacional em contextos hospitalares. Revista Prática Hospitalar, São Paulo, v. 3, n. 43, p. 158-164, 2006.

ESQUENAZI, D.; SILVA, S. R. B.; GUIMARÃES, M. A. M. Aspectos fisiopatológicos do envelhecimento humano e quedas em idosos. Revista Hospital Universitário Pedro Ernesto, Rio de Janeiro, v. 13, n. 2, p. 11-20, 2014.

GALHEIGO, S. M. Domínios e temáticas no campo das práticas hospitalares em terapia ocupacional: uma revisão da literatura brasileira de 1990 a 2006. Revista de Terapia Ocupacional da Universidade de São Paulo, São Paulo, v. 18, n. 3, p. 113-121, 2007.
GALHEIGO, S. M.; ANGELI, A. A. C. Terapia Ocupacional e o cuidado integral à saúde de crianças e adolescentes: a construção do Projeto Accalanto. Revista de Terapia Ocupacional da Universidade de São Paulo, Sáo Paulo, v. 18, n. 1, p. 10-15, 2008.

MARTINS, R. F. et al. Aspectos estruturais do ambulatório de Terapia Ocupacional na área de infância e adolescência da rede de reabilitaçáo Lucy Montoro Unidade Ribeirăo - HCFMRP - USP. In: UCHÔA-FIGUEIREDO, L. R.; NEGRINI, S. F. B. M. Terapia Ocupacional: diferentes práticas em hospital geral. Ribeiráo Preto: Legis Summa, 2009. p. 107-116.

PENGO, M. M. S. B. Contribuiçóes da Terapia Ocupacional na assistência da mulher mastectomizada no Hospital Amaral Carvalho de Jaú. Revista CETO, São Paulo, v. 13, n. 13, p. 72-84, 2012.

RABELO, L. P. P. et al. Perfil de idosos internados em um hospital universitário. Revista Mineira de Enfermagem, Belo Horizonte, v. 14, n. 3, p. 293-300, 2010.

SANTOS, C. A. V.; DE CARLO, M. M. R. P. Hospital como campo de práticas: revisão integrativa da literatura e a terapia ocupacional. Cadernos de Terapia Ocupacional da UFSCar, São Carlos, v. 21, n. 1, p. 99-107, 2013.

SILVEIRA, A. M.; JOAQUIM, R. H. V. T.; CRUZ, D. M. C. Tecnologia Assistiva para a promoção de atividades da vida diária com crianças em contexto hospitalar. Cadernos de Terapia Ocupacional da UFSCar, São Carlos, v. 20, n. 2, p. 183-190, 2012.

TENÓRIO, D. M.; CAMACHO, A. C. L. F. Identificação dos agravos de saúde que levam os idosos ao serviço de emergência. Revista de Enfermagem UFPE, Recife, v. 9, p. 457-465, 2015. Suplemento 1.

\section{Contribuição dos Autores}

Miryam Bonadiu Pelosi e Janaína Santos Nascimento foram responsáveis pela concepção do texto, organização de fontes e análises, redação e revisão do artigo. Todos os autores aprovaram a versão final do texto.

\section{Fonte de Financiamento}

CNPq: 476378/2011-7 - Edital UNIVERSAL.

\section{Notas}

${ }^{1}$ Este estudo integra o projeto de pesquisa: "Comparando Recursos da Comunicação Alternativa para Pacientes Hospitalizados com Dificuldades de Fala”. 